\title{
Modification of the CdTe-In Interface by Irradiation with Nanosecond Laser Pulses through the CdTe Crystal
}

\author{
Kateryna S. ZELENSKA*1, Dmytro V. GNATYUK ${ }^{* 2}$ and Toru AOKI ${ }^{* 3,4}$ \\ ${ }^{* 1}$ Taras Shevchenko National University of Kyiv, 64/13 Volodymyrska Str., Kyiv 01601, Ukraine \\ E-mail: czelenska@gmail.com \\ ${ }^{* 2}$ Graduate School of Science and Technology, Shizuoka University, 3-5-1 Johoku, Naka-ku, \\ Hamamatsu 432-8011, Japan \\ ${ }^{* 3}$ Graduate School of Informatics, Shizuoka University, 3-5-1 Johoku, Naka-ku, \\ Hamamatsu 432-8011, Japan \\ ${ }^{* 4}$ Research Institute of Electronics, Shizuoka University, 3-5-1 Johoku, Naka-ku, \\ Hamamatsu 432-8011, Japan
}

\begin{abstract}
Laser processing of the CdTe-In semiconductor-metal interface has been studied by irradiation through the CdTe crystal in water using nanosecond pulses with $\lambda=1064 \mathrm{~nm}$. A thin In-doped CdTe layer adjacent to the In film was formed after direct laser impact on the CdTe-In interface. The fabricated $\mathrm{In} / \mathrm{CdTe} / \mathrm{Au}$ diode detectors with a $p-n$ junction exhibited steep rectification and sensitivity to $\mathrm{X} / \gamma$-ray radiation. The simulation of laser-induced heating of the In film irradiated through the $\mathrm{CdTe}$ in water was performed. Temperature distribution inside the three-layer CdTe-In-Water structure at different moments under the action of nanosecond laser pulse was calculated.
\end{abstract}

DOI: $10.2961 /$ jlmn.2015.03.0011

Keywords: pulsed laser irradiation, laser-induced doping, temperature distribution, metalsemiconductor interface, $p-n$ junction, diode structure, $I-V$ characteristic, $\mathrm{X} / \gamma$-ray detector

\section{Introduction}

Key problems in development and fabrication of semiconductor devices are modification of the surface and interface states, transformation of the structure and purposeful change of parameters of a thin surface region of semiconductor crystals. Moreover, the applied procedures should not alter the structure and properties of the bulk of crystals. In this case, laser processing has been advantageously employed for modification of semiconductor crystal characteristics and formation of various kinds of semiconductorbased device structures [1-3]. Application of short laser pulses to treat semiconductors with radiation strongly absorbed by a thin surface layer allows to avoid disturbance of the structure in the crystal volume and to form thin layers and interfaces with desired characteristics.

Nanosecond laser processing is widely used to change the surface state, structure and parameters of surface layers of CdTe semiconductor which is the basic material for solid state high energy radiation detectors [4-13]. In particular, nanosecond laser pulses have been successfully employed for the processing of CdTe-In structures to modify the metal-semiconductor interface, form a thin heavy doped layer and create a built-in electrical junction [8-13].

The ability of CdTe diode structures to detect and effectively perform energy discrimination of $\mathrm{X} / \gamma$-ray radiation is greatly important property that is used in detection instruments employed in industry, science, medicine, security, environment protection, etc. [14-17]. In order to obtain diode type CdTe-based detectors with a built-in $p$ - $n$ junction, the laser-induced doping of a thin surface layer of the semiconductor has been developed [9-13]. It allowed us to suppress dopant self-compensation thus, to introduce and activate In impurity atoms in the surface region of semiinsulating CdTe crystals. The method consisted of irradiation of high resistivity $p$-like CdTe crystals pre-coated with an In dopant film by nanosecond laser pulses from the In side [9-13]. The best characteristics of M- $p-n \mathrm{In} / \mathrm{CdTe} / \mathrm{Au}$ diode detectors were obtained when the CdTe-In structures were irradiated in water [11-13].

The doping mechanism was attributed with the action of a laser-induced stress wave which penetrated through the relatively thick In film, affected the In-CdTe interface and transformed to a shock wave [10]. Despite the advantages of such laser-induced solid phase doping, direct laser irradiation of the dopant film resulted in (1) evaporation of In, (2) nonuniform generation and propagation of stress and shock waves and (3) it was not possible to directly influence the CdTe-In interface [9-13].

In this work, we suggest the advanced technique of laser-induced doping by irradiation of the CdTe-In structures from the CdTe side. For this purpose, nanosecond pulses of the first harmonic $(\lambda=1064 \mathrm{~nm})$ of a YAG:Nd laser were used. CdTe is transparent for this radiation wavelength and it is possible to irradiate the CdTe-In structure through the CdTe crystal. It allowed affecting directly the CdTe-In interface because irradiation is strongly absorbed by a thin layer of the In film. Extreme conditions in the confined area at the CdTe-In interface under laser irradiation resulted in the formation of a diode structure with a high barrier $p-n$ junction. The simulation of laser heating of the three-layer CdTe-In-Water structure has been performed. This approach fundamentally differs from the calculations of the temperature distribution in CdTe crystals under laser irradiation $[18,19]$. 


\section{Experimental details}

\subsection{Preparation of samples}

Experimental investigations were carried out using commercial detector grade CdTe single crystals grown by the traveling heater method from Acrorad Corporation [14]. The room temperature resistivity of the semiconductor was $\rho=(4-6) \times 10^{9} \Omega \cdot \mathrm{cm}$ that was close or even higher than the intrinsic value. Parallelepiped-like (111) oriented CdTe wafers had the area of $5 \times 5 \mathrm{~mm}^{2}$ and thickness of $0.5 \mathrm{~mm}$ or $1 \mathrm{~mm}$. Before doping and electrode deposition, the CdTe wafers were subjected to preliminary surface processing. Initially polished CdTe crystals obtained from the manufacturer were cleaned in acetone and methanol, subjected to chemical polishing etching in $5 \%$ bromine in methanol solution and then the samples were thoroughly rinsed in methanol. The procedures of the sample preparation were similar to ones used before [9-13].

A relatively thick $(0.3-2.00 \mu \mathrm{m})$ In dopant film was evaporated on the $\mathrm{CdTe}(111) \mathrm{B}$ crystal surface (Teterminated face) in a physical vapor deposition system at low pressure without heating the samples. The In film served as an $n$-type dopant source during laser irradiation and also as an electrode after laser-induced doping. Schematic illustration of the $\mathrm{CdTe}(111)$ crystalline structure and investigated sample was shown in [11].

In our previous works, laser-induced doping was carried out by irradiation of CdTe-In structures from the In film side with nanosecond laser pulses of different lasers in various environments (vacuum, ambient air, under argon pressure and water). In all the cases, laser irradiation was strongly absorbed by a thin $(\sim 10 \mathrm{~nm})$ surface In layer and solid phase doping of the CdTe surface region was associated with the effect of stress and shock waves which were generated as a result of fast heating, melting, and evaporation of a thin In layer [11-13]. It was established that the use of the liquid (water) environment provided the conditions for higher stress and shock wave amplitudes and the deposited In film was not completely evaporated and destroyed even at multiple irradiation of CdTe-In structures with laser pulses of high energy densities [11-13].

\subsection{Advanced technique of laser-induced doping by irradiation of CdTe-In structures from the CdTe side}

The key feature of the proposed technique of laserinduced doping of a thin layer of CdTe crystals and formation of a $p-n$ junction consisted of the direct impact of laser pulses on the semiconductor-metal interface. For this purpose, the first harmonic of a YAG:Nd laser $(\lambda=1064$ $\mathrm{nm})$ was employed. CdTe semiconductor is transparent for this radiation wavelength and it was possible to irradiate the CdTe-In structure through the CdTe crystal to directly heat a thin layer of the In film and affect the interface. The laser emitted nanosecond pulses $(\tau=8 \mathrm{~ns})$ with repetition rate $10-12 \mathrm{~Hz}$. The pulse energy density was varied in the range $100-800 \mathrm{~mJ} / \mathrm{cm}^{2}$. At such laser parameters, samples cooled between pulses, hence the heat accumulation effect in irradiated samples was negligible.

The schematic illustration of the technological procedures of the fabrication of M- $p-n \mathrm{In} / \mathrm{CdTe} / \mathrm{Au}$ structured diodes is shown in Fig. 1. The whole area $\left(5 \times 5 \mathrm{~mm}^{2}\right)$ of the CdTe-In structures was entirely irradiated from the
CdTe side with nanosecond pulses in distilled water at room temperature (Fig. 1(b)). The laser radiation passed through the CdTe crystal and was strongly absorbed by a thin layer of the In film. Therefore it was possible to impact directly the CdTe-In interface. Water as was used as the unloading media to provide output for stress and shock waves generated in solids under laser action.

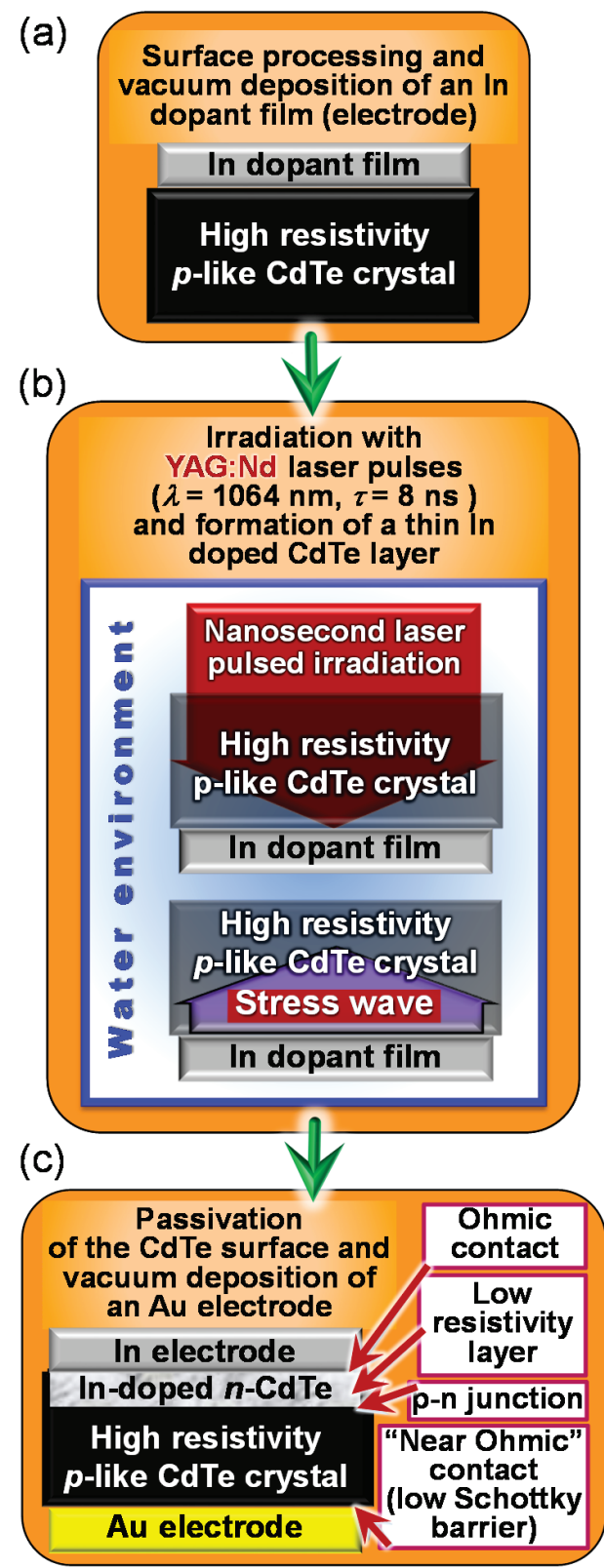

Fig.1 The procedures of fabrication of $\mathrm{In} / \mathrm{CdTe} / \mathrm{Au}$ diode detectors with a $p$ - $n$ junction: surface processing of the $p$-like CdTe crystal and deposition of an In dopant (electrode) film (a); laserinduced doping of a thin CdTe layer by irradiation of the CdTe-In interface through the CdTe in water (b); passivation of the CdTe surface, deposition of an Au electrode and schematic illustration

of the formed multilayered M- $p-n \mathrm{In} / \mathrm{CdTe} / \mathrm{Au}$ diode (c).

Energy density and number of laser pulses were varied in wide ranges to find the optimal laser processing regimes which provided the effective doping of a thin CdTe layer adjoining to the In film.

After laser irradiation of the CdTe-In structures and prior to deposition of an $\mathrm{Au}$ electrode, the samples were 
dipped into an aqueous $\mathrm{H}_{2} \mathrm{O}_{2}$ solution and then rinsed in methanol. An Au contact was similarly evaporated in vacuum on the opposite side of the samples, i.e. on the CdTe(111)A surface (Cd-terminated) (Fig. 1(c)). The thickness of the Au electrode was about $0.5 \mu \mathrm{m}$. Hydrogen peroxide passivation was applied for modification of the surface state of the CdTe before forming an Au contact, decrease of the lateral surface component of leakage current and stabilization of electrical properties of the $\mathrm{In} / \mathrm{CdTe} / \mathrm{Au}$ diodes. Moreover, when In and Au were evaporated in vacuum on the CdTe surfaces, a mask was used to prevent ingress of the metals on the lateral sides of the samples. The In and $\mathrm{Au}$ electrodes, formed on the polar faces of the CdTe(111) crystal, had the area of $4 \times 4 \mathrm{~mm}^{2}$ (Fig. 1(c)).

\subsection{Measurement techniques of $\mathrm{In} / \mathrm{CdTe} / \mathrm{Au}$ diodes}

The fabricated $\mathrm{In} / \mathrm{CdTe} / \mathrm{Au}$ diode detector was a complex metal-semiconductor structure: In electrode, $\mathrm{In} / n$ CdTe Ohmic contact, thin low resistivity heavily doped $n$ CdTe layer, $p-n$ junction, bulk part of semi-insolating $p$-like CdTe, Au/CdTe low Schottky barrier (near Ohmic contact) and $\mathrm{Au}$ electrode (Fig. 1(c)).

The obtained M- $p-n \operatorname{In} / \mathrm{CdTe} / \mathrm{Au}$ diodes were tested by electrical and spectral measurements. $I-V$ characteristics of the diodes were studied in the dark condition at room temperature. The forward current was measured when the Au electrode was positively biased with respect to the In electrode. The energy spectra of a ${ }^{137} \mathrm{Cs}$ radioisotope taken by the $\mathrm{In} / \mathrm{CdTe} / \mathrm{Au}$ diode detectors were measured at applied reverse bias voltage $V=100-400 \mathrm{~V}$ at room temperature.

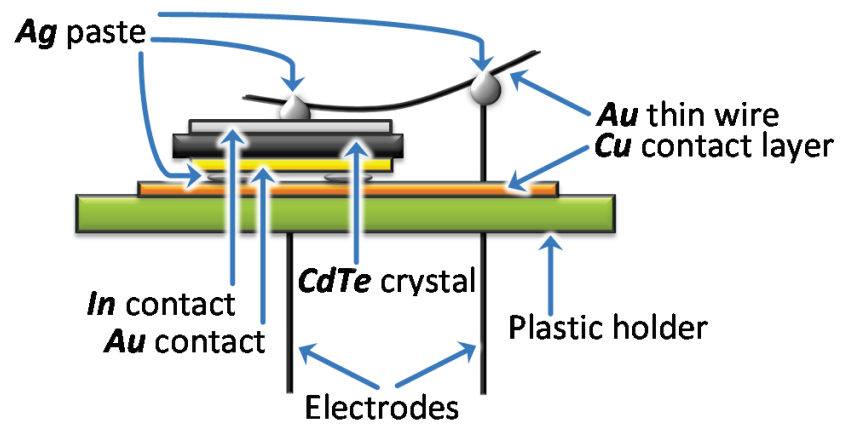

Fig. 2 Schematic illustration of the $\mathrm{In} / \mathrm{CdTe} / \mathrm{Au}$ diode detector placed on the plastic holder and connected to electrodes for electrical and spectral measurements.

In order to perform the electrical and spectral measurements, the $\mathrm{In} / \mathrm{CdTe} / \mathrm{Au}$ diodes were placed on the holder and In and $\mathrm{Au}$ contacts were connected to the external electrodes using silver paste and a fine gold wire (Fig. 2).

\section{Experimental results and discussion}

\subsection{Electrical characteristics of $\mathrm{In} / \mathrm{CdTe} / \mathrm{Au}$ diodes}

An $I-V$ characteristic is one of the most significant parameters of a diode. Fig. 3 shows the typical $I-V$ characteristics of the fabricated $\mathrm{In} / \mathrm{CdTe} / \mathrm{Au}$ structures before (curve 1 ) and after (curves 2 and 3 ) laser action. The $I-V$ characteristic of the unirradiated $\mathrm{In} / \mathrm{CdTe} / \mathrm{Au}$ sample exhibited rectifying properties that was due to influence of a Schottky contact of CdTe-In (Fig. 3, curve 1). Multiple irradiation of the CdTe-In interface through the CdTe increased for- ward-biased current and decreased reverse one (Fig. 3, curves 2 and 3 ).

The $I-V$ characteristic profile depended on energy density of incident laser radiation and number of laser shots as well as on the thickness of the deposited In electrode film. Irradiation of the $\mathrm{In} / \mathrm{CdTe} / \mathrm{Au}$ structures from the $\mathrm{CdTe}$ crystal side remarkably shifted the forward branch of the $I$ $V$ curve toward lower voltages and reduced the leakage current $(I=12 \mathrm{nA}$ and $I=1.2 \mathrm{nA}$ at $V=200 \mathrm{~V}$ for curves 2 and 3 , respectively) in comparison with an unirradiated $\mathrm{In} / \mathrm{CdTe} / \mathrm{Au}$ structure $(I=44 \mathrm{nA}$ at $V=200 \mathrm{~V}$, curve 1$)$.

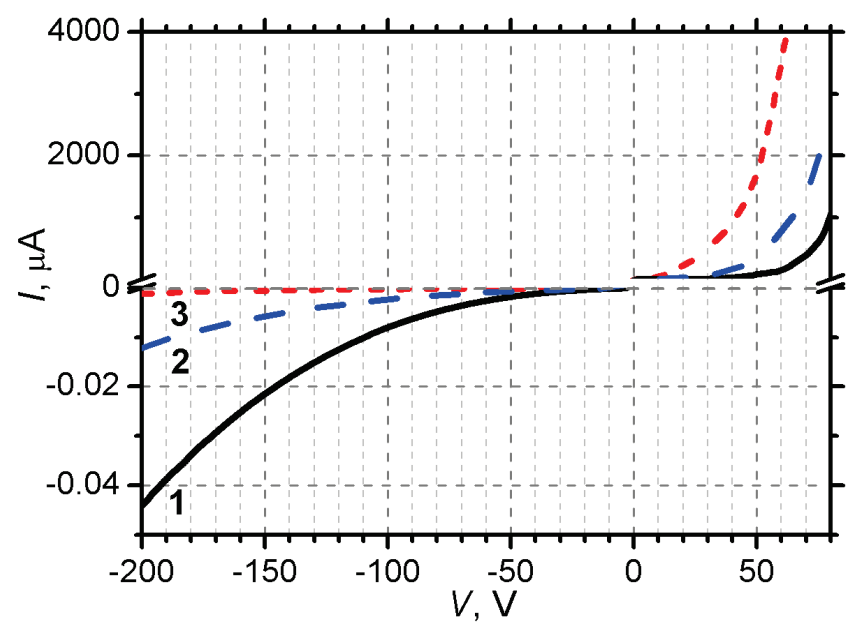

Fig. 3 Room temperature $I-V$ characteristics of the $\mathrm{In} / \mathrm{CdTe} / \mathrm{Au}$ structures before (1) and after laser irradiation of the CdTe-In interface through the CdTe crystal with 10 pulses of energy density of $424 \mathrm{~mJ} / \mathrm{cm}^{2}$ (curve 2) and 50 pulses of $144 \mathrm{~mJ} / \mathrm{cm}^{2}$ (curve

3). The In dopant (electrode) film thickness was $0.5 \mu \mathrm{m}$.

A low leakage current and a steep increase in the forward biased current were evidence of the creation of a high barrier $p-n$ junction in the CdTe crystal near the CdTe-In interface. This was due to the formation of a heavily Indoped thin $n$-type CdTe layer adjoining to the CdTe-In interface as result of laser action on the In film.

One of the peculiarities of the $I-V$ characteristics of the $\mathrm{In} / \mathrm{CdTe} / \mathrm{Au}$ diodes fabricated by laser-induced doping with irradiation of the CdTe-In through CdTe in water was very high rectification, especially high forward current at relative low leakage current (Fig. 3, curve 3).

\subsection{Spectral characteristics of In/CdTe/Au detectors}

The performance of the fabricated $\mathrm{In} / \mathrm{CdTe} / \mathrm{Au}$ diode detectors with a $p$ - $n$ junction was tested for high energy radiation detection. Fig. 4 shows the ${ }^{137} \mathrm{Cs}$ radioisotope energy spectrum taken by the $\mathrm{In} / \mathrm{CdTe} / \mathrm{Au}$ detector at room temperature and applied bias voltage $V=200 \mathrm{~V}$. The energy resolution (full width at half maximum, FWHM) of majority samples with low leakage current at room temperature was FWHM $=6-8 \%$ at the $662 \mathrm{keV}$ peak at applied reverse bias voltage of 100-300 V. The value of FWHM was not so high, however the $\mathrm{In} / \mathrm{CdTe} / \mathrm{Au}$ diodes demonstrated enough sensitivity to $\mathrm{X} / \gamma$-ray radiation to be employed for detection devices.

Certainly, we have obtained higer energy resolution using laser-induced doping with pulsed laser irradiation of CdTe-In structures from the In film side $[11,13]$. However, the modified technique with the use of laser radiation, 
which passes through the CdTe and then is absorbed by a thin layer of the In film thus, directly impacts the CdTe-In interface, shows promise and needs future investigation. One of the interesting features of the applied method is the time stability of functional parameters of the fabricated $\mathrm{In} / \mathrm{CdTe} / \mathrm{Au}$ diode detectors.

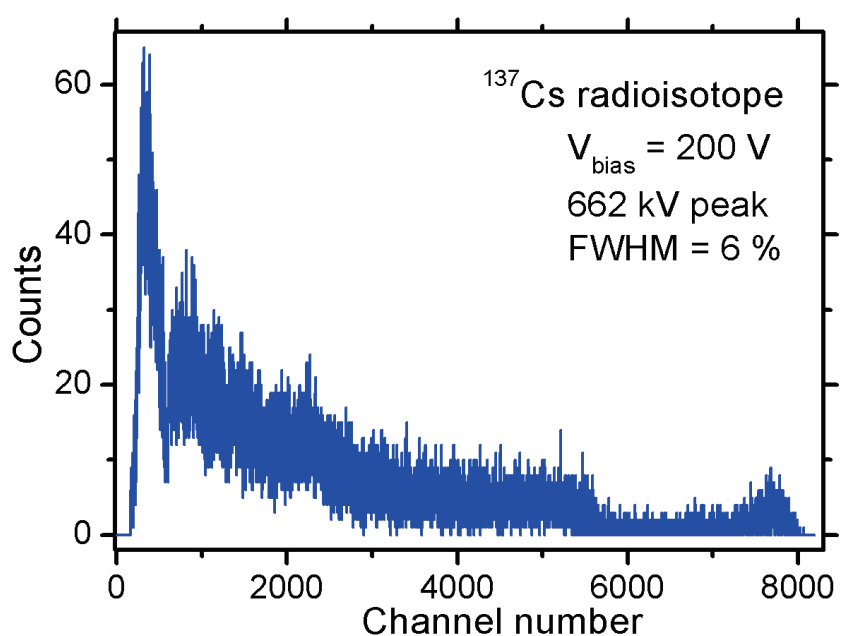

Fig. 4 Room temperature energy spectrum of a ${ }^{137} \mathrm{Cs}$ radioisotope taken by the $\mathrm{In} / \mathrm{CdTe} / \mathrm{Au}$ diode detector with a $p$ - $n$ junction formed by the laser-induced doping with laser irradiation of the CdTe-In interface through the CdTe crystal in water.

\section{Simulation of laser heating of three layer CdTe-In- Water structure}

To study the features of physical mechanisms of laserinduced action on the three layer CdTe-In-Water structure, processes of doping and creation of a $p-n$ junction in the $p$ like CdTe near the CdTe-In interface under irradiation of the CdTe-In interface through the CdTe crystal, the laser heating simulation was performed.

A three-layer structure model was considered: semiinfinite CdTe semiconductor crystal, In film with a certain thickness and semi-infinite water layer. Temperature distributions inside all the layers were estimated for different laser energy densities and thicknesses of the In dopant film. The calculation was based on the thermal conduction equation considering a laser pulse as a Gaussian beam $[18,19]$. The thermal and optical parameters of CdTe, In and water were taken into account as temperature independent ones $[16,17,20]$. In particular, water and CdTe were assumed as transparent media for the chosen laser wavelength (1064 $\mathrm{nm}$ ) and radiation was absorbed by only a thin In layer adjoining to the CdTe crystal.

The characteristics of the $\mathrm{In} / \mathrm{CdTe} / \mathrm{Au}$ diode detectors with a $p-n$ junction, formed by laser irradiation of the CdTe-In interface through the CdTe crystal in water depended on the thickness of the deposited In dopant (electrode) film, energy density and number of laser pulses. The diodes exhibited steep rectification properties and sensitivity to $\mathrm{X} / \gamma$-ray radiation in the cases of a thin $(0.3-0.5 \mu \mathrm{m})$ or thicker (up to $2 \mu \mathrm{m}$ ) In film after action of certain number of laser pulses with corresponding energy densities.

The calculated temperature distribution inside the CdTe-In-Water structure for the In film thickness of $0.5 \mu \mathrm{m}$ and laser pulse energy density of $144 \mathrm{~mJ} / \mathrm{cm}^{2}$ is shown in Fig. 5. The numbered curves demonstrate the temperature distribution in the thicknesses $Z$ of three media (CdTe, In and water) at different moments $t$ after the laser pulse with duration of $8 \mathrm{~ns}$ has reached its amplitude: initial moment (curve 1) and after $4 \mathrm{~ns}$ (curve 2), 6 ns (curve 3), $8 \mathrm{~ns}$ (curve 4), 10 ns (curve 5) and 56 ns (curve 6).

The temperatures of the front and back In film surfaces differ by 10-30 Kelvin and rise in 0-6 ns (Fig. 5, curves 12 ). The temperature distribution becomes uniform within the In film and decreases after the end of laser pulse action $(8 \mathrm{~ns})$ as seen from the curves $4-6$ for $t=8 \mathrm{~ns}, t=10 \mathrm{~ns}$ and $t=56 \mathrm{~ns}$ respectively.

The calculated time dependence of the temperature of the front (curve 1) and back (curve 2) surfaces of the In dopant film irradiated through the CdTe side by a laser pulse with power density of $144 \mathrm{~mJ} / \mathrm{cm}^{2}$ is shown in Fig. 6 . The simulated shape of a laser pulse is shown with the dashed curve. The temperature of the front surface almost reaches the melting point of $\operatorname{In}\left(T_{\mathrm{m}}=429 \mathrm{~K}\right)$ due to strong absorption of laser radiation by a thin In layer adjoining to the CdTe [20]. It should be mentioned that the temperatures of the front and back In film surfaces rise with different rate under the laser pulse action as well as these temperatures decrease simultaneously when the uniform temperature distribution occurs after the end of laser pulse (Fig. 5).

As seen from the calculation results, irradiation of the CdTe-In interface through the CdTe crystal in water with laser pulse energy density of $144 \mathrm{~mJ} / \mathrm{cm}^{2}$ resulted in heating the entire thin $(0.3-0.5 \mu \mathrm{m})$ In dopant film up to the melting point (Fig. 5, curves 3-5). Good characteristics of the $\mathrm{In} / \mathrm{CdTe} / \mathrm{Au}$ diode detectors were also achieved when the thick (about $2 \mu \mathrm{m}$ ) In film was used. In this case, an In layer near the CdTe-In interface was under the melting temperature while the external In film surface (adjoining to water) remained at room temperature.

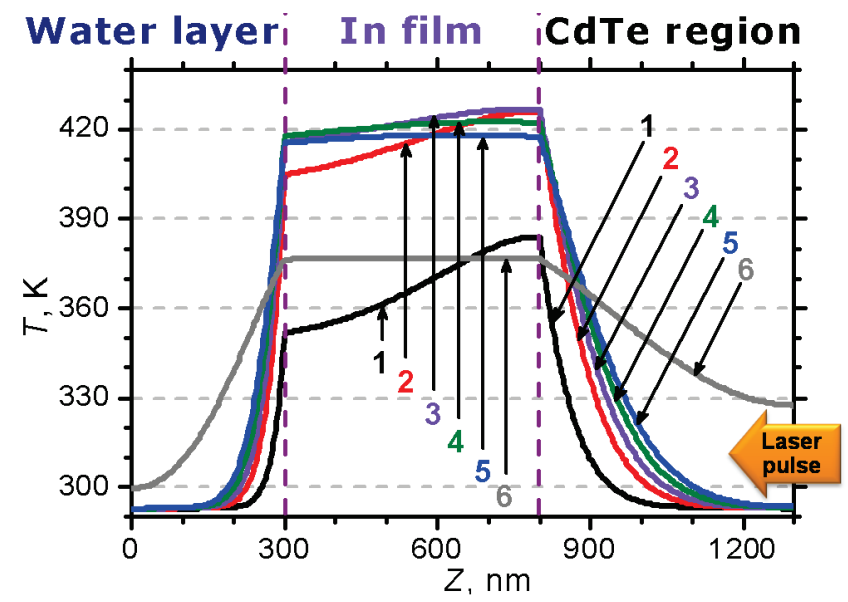

Fig. 5 The calculated temperature distributions inside the CdTeIn-Water structure under irradiation of the CdTe-In interface through the CdTe crystal in water with a single pulse of a YAG:Nd laser ( $\lambda=1064 \mathrm{~nm}, \tau=8 \mathrm{~ns})$ of energy density of 144 $\mathrm{mJ} / \mathrm{cm}^{2}$ at different moments $t$ after the laser pulse has reached its maximum: 0 ns (curve 1), 4 ns (curve 2), 6 ns (curve 3), 8 ns (curve 4), 10 ns (curve 5), 56 ns (curve 6). Axis $\mathrm{Z}$ indicates the thicknesses of the In film and also CdTe region and water layer adjoined to the film.

The simulation of heating processes in the three layer CdTe-In-Water structure and calculation of temperatures in the vicinity of the CdTe-In interface can be developed and 
used to find the optimal regimes (In film thickness and laser irradiation parameters) for the effective In doping of a thin CdTe layer near the CdTe-In interface under laser irradiation of the In dopant film through the CdTe crystal.

Despite the fact that the CdTe-In interface was heated to not so high temperature (near the melting point of In), such fast adiabatic heating of a thin laser radiationabsorbing layer of the In dopant film resulted in a very significant temperature gradient $\left(10^{10} \mathrm{~K} / \mathrm{s}\right)$. This evoked rapid thermal expansion of the near-interface In layer and caused a very considerable stress gradient, resulting in the generation of a high amplitude stress wave. The pressure in the initial front of a stress was about $50 \mathrm{MPa}$ [10].

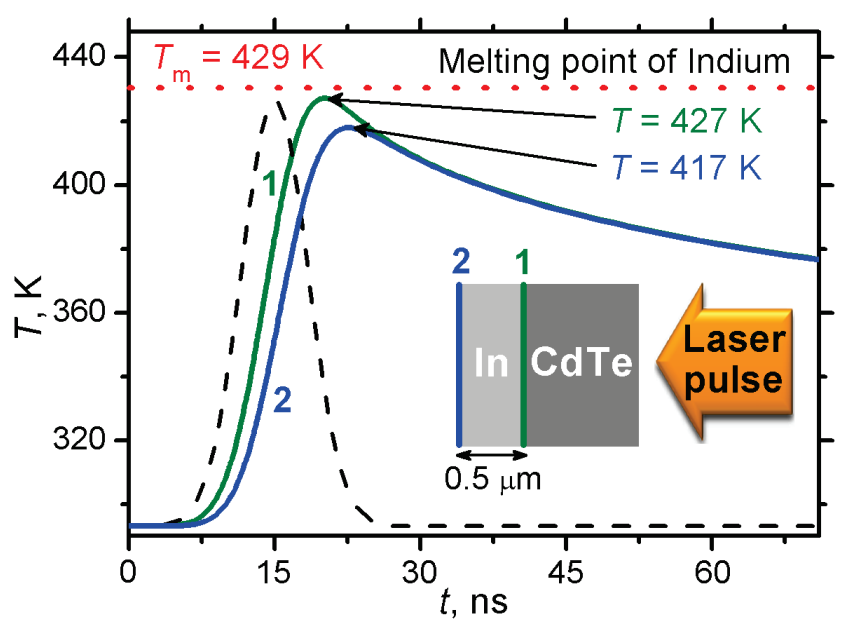

Fig. 6 The calculated time dependences of the temperature of the front (curve 1) and back (curve 2) faces of the In dopant (electrode) film pre-deposited on the CdTe crystal. The melting point of In is indicated by the dotted line. The laser pulse profile is shown with the dashed curve.

Indium atoms implicated by the laser-induced stress wave and penetrated into a thin CdTe layer in the vicinity of the CdTe-In interface. In the high stress field, a large number of cadmium vacancies $\mathrm{V}_{\mathrm{Cd}}$ can be created as result of desorption of $\mathrm{Cd}$ atoms from the crystal lattice sites and dissociation of $\mathrm{V}_{\mathrm{Cd}^{-}} \mathrm{X}$ complexes [12]. Coefficients of segregation and diffusion of an In donor impurity significantly increased under a powerful compression pulse which was laser-generated at the CdTe-In interface and being propagated into the CdTe bulk $[16,17]$. Moreover, a stressinduced increase in a number of $\mathrm{V}_{\mathrm{Cd}}$ in CdTe resulted in drastically enhanced diffusion of In atoms at $\mathrm{Cd}$ vacancies to ensure the penetration of In atoms in the CdTe crystal. Indium atoms, incorporated in a CdTe crystal by the laserinduced stress wave, substituting $\mathrm{Cd}$ atoms acted as donors. A steep In profile in the CdTe region near the CdTe-In interface formed a shallow built-in $p-n$ junction.

\section{Conclusion}

Irradiation of high resistivity $p$-like CdTe crystals precoated with an In dopant film from the CdTe side by nanosecond laser pulses with wavelength that is not absorbed by the semiconductor made it possible to directly affect the CdTe-In interface because radiation was strongly absorbed by a thin layer of the In film adjoining to the CdTe crystal. The doping mechanism was associated with the action of laser-induced stress wave which was generated under ex- treme conditions in the confined area at the CdTe-In interface under laser irradiation. The developed technique allowed avoiding evaporation of In dopant and resulted in the formation of the In-doped CdTe region and thus, creation of a built-in $p-n$ junction. The temperature distribution inside the three layer CdTe-In-Water structure was calculated and correlations between the characteristics of the fabricated $\mathrm{In} / \mathrm{CdTe} / \mathrm{Au}$ diodes and laser processing conditions were obtained.

\section{Acknowledgments}

This research was partly supported by the NATO Science for Peace and Security Programme. Grant No NUKR.SFPP 984705 - A Sensor Network for the Localization and Identification of Radioactive Sources (SENERA).

\section{References}

[1] "Laser Annealing of Semiconductors" ed. by J. M. Poate and J. W. Mayer, (Academic Press, New York, 1982) p.576.

[2] R. T. Young: "Pulsed Laser Processing of Semiconductors", in "Semiconductors and Semimetals" ed. by R. F. Wood, C. W. White and R. T. Young, (Academic Press, New York, 1985) p.693.

[3] "Resent Advances in Laser Processing of Materials" ed. by J. Perriere, E. Millon and E. Fogarassy, (Elsevier, Oxford, 2006) p.472.

[4] V. A. Gnatyuk, T. Aoki, Y. Nakanishi and Y. Hatanaka: Surf. Sci., 542, (2003) 142.

[5] T. Aoki, D. V. Gnatyuk, V. A. Odarych, L. V. Poperenko, I. V. Yurgelevych and S. N. Levytskyi: Thin Solid Films, 519, (2011) 2834

[6] A. Medvid, A. Mychko, E. Dauksta, V. Ivanov, L. Alekseeva, E. Dieguzs, J. Crosso and H. Bensalah: Nucl. Sci. Symp. Conf. Rec. (NSS/MIC), IEEE, (2012) 4672.

[7] D. V. Gnatyuk, L. V. Poperenko, I. V. Yurgelevych, O. I. Dacenko and T. Aoki: IEEE Trans. Nucl. Sci., 62, (2015) 428.

[8] J. L. Shaw, R. E. Viturro, L. J. Brillson, D. Kilday and G. Margaritondo: J. Electron. Mater., 17, (1988) 149.

[9] Y. Hatanaka, M. Niraula, A. Nakamura, T. Aoki and Y. Nakanishi: Appl. Surf. Sci., 142, (1999) 227.

[10] V. A. Gnatyuk, T. Aoki and Y. Hatanaka: Appl. Phys. Lett., 88, (2006) 242111.

[11] V. A. Gnatyuk, T. Aoki, O. I. Vlasenko and S. N. Levytskyi: Nucl. Sci. Symp. Conf. Rec. (NSS/MIC), IEEE, (2011) 4506.

[12] V. A. Gnatyuk, S. N. Levytskyi, O. I. Vlasenko and T. Aoki: Adv. Mater. Res., 222, (2011) 32.

[13] V. A. Gnatyuk, T. Aoki, E. V. Grushko, L. A. Kosyachenko, and O. I. Vlasenko: Proc. SPIE, 8142 (2011) 81420B.

[14]H. Shiraki, M. Funaki, Y. Ando, S. Kominami, K. Amemiya and R. Ohno: IEEE Trans. Nucl. Sci., 57, (2010) 395.

[15] S. Del Sordo, L. Abbene, E. Caroli, A. M. Mancini, A. Zappettini and P. Ubertini: Sensors, 9, (2009) 3491.

[16] K. Zanio: "Cadmium telluride", in "Semiconductors and Semimetals" ed. by K. Willardson and A. C. Beer, (Academic Press, New York, 1978) p.235. 
[17] S. Adachi: "Properties of Semiconductor Alloys: Group-IV, III-V and II-VI Semiconductors" ed. by: P. Capper, S. Kasap and A. Willoughby, (Wiley, Chippenham, 2009) p.422.

[18] V. A. Gnatyuk, T. Aoki, O. S. Gorodnychenko and Y. Hatanaka: Appl. Phys. Lett., 83, (2003) 3704.
[19] S. P. Zhvavyi and G. L. Zykov: Semiconductors, 40, (2006) 632.

[20] R. Y. Koyama, N. V. Smith and W. E. Spicer: Phys. Rev. B, 8, (1973) 2426.

(Received: June 5, 2015, Accepted: November 13, 2015) 